livraisons

d'Histoire

de l'Architecture

\section{Livraisons de l'histoire de l'architecture}

18 | 2009

D'architectures

\title{
L'hôtel Choudens
}

Music Editor Chouden's townhouse

Das Hôtel Choudens

\section{Agnès Chauvin}

\section{CpenEdition}

\section{Journals}

Édition électronique

URL : http://journals.openedition.org/lha/224

DOI : 10.4000/lha.224

ISSN : 1960-5994

Éditeur

Association Livraisons d'histoire de l'architecture - LHA

Édition imprimée

Date de publication : 10 décembre 2009

Pagination : 65-76

ISSN : $1627-4970$

Référence électronique

Agnès Chauvin, "L'hôtel Choudens », Livraisons de l'histoire de l'architecture [En ligne], 18| 2009, mis en ligne le 10 décembre 2011, consulté le 04 mai 2019. URL : http://journals.openedition.org//ha/224 ; DOI : 10.4000/lha.224

Ce document a été généré automatiquement le 4 mai 2019.

Tous droits réservés à l'Association LHA 


\title{
L'hôtel Choudens
}

\author{
Music Editor Chouden's townhouse \\ Das Hôtel Choudens
}

Agnès Chauvin

1 Dans les dernières années du XIX siècle et le tout début du XXe siècle, les aristocrates et les grands bourgeois de la finance souhaitent faire disposer, dans la construction de leurs demeures, de partis stylistiques plus conformes à leurs goûts ainsi que du confort apporté par les progrès des dernières innovations techniques.

\section{Les Choudens, une famille d'éditeurs de musique}

2 Originaire de Genève, et petit-fils de l'éditeur musical Antoine Pacini, Antoine de Choudens (1825-1888) fonde une maison d'édition musicale en 1844. Il oriente le tout premier répertoire de la maison vers l'art vocal italien avec, semble-t-il, les premières années, une production assez faible ${ }^{1}$. En revanche, l'acquisition en 1859, pour une somme de 10000 francs, du manuscrit de Faust de Berlioz refusé par la maison Heugel, appartient désormais à la légende. Antoine de Choudens a aussi l'intuition de publier deux œuvres qui compteront parmi les plus importants opéras français du XIX siècle : Les Troyens en 1863 et Carmen en 1875. Ce coup de génie lui permet d'acquérir les fonds de trois autres maisons et de faire naître, en 1845, avec ses deux fils, Antony et Paul, une maison d'une envergure jamais vue.

3 Dirigées par Paul à partir de 1892, les éditions poursuivent la publication de nombreuses œuvres lyriques et des premières pièces pour piano de Debussy². La maison Choudens prospère et acquiert alors une notoriété incomparable.

\section{Le choix d'un quartier et d'un style architectural}

4 Paul de Choudens (1850-1925) habitait la rue de Milan. Pour faire construire son hôtel particulier, il reste fidèle au $9^{\mathrm{e}}$ arrondissement et choisit d'élire domicile dans le quartier 
de la Nouvelle Athènes. Celui-ci, légèrement en déclin depuis la troisième République, a gardé beaucoup de son pouvoir d'attraction pour le milieu musical. La rue Blanche, proche de sa maison d'édition ${ }^{3}$ et de nombreuses salles de concert, sent « l'opulence et la vie correcte ». S'il est possible d'expliquer le choix de ce quartier, il est plus difficile de savoir pour quelles raisons Paul de Choudens a fait appel à Charles Girault. Cet architecte, comme ses confrères Louis Parent ou René Sergent, s'est spécialisé dans un style qui fait la transition entre le Louis XV et le Louis XVI et qui plait beaucoup aux commanditaires.

En septembre 1899, la veuve d'Arthur Péricault de Gravillon vend à Paul de Choudens son petit hôtel, d'un étage, entre cour et jardin situé 21 , rue Blanche ${ }^{4}$. L'habitation est détruite et le 23 mai 1901, l'architecte Charles Girault dépose le permis de construire d'un hôtel particulier au nom de Paul de Choudens ${ }^{5}$ (ill. 1).

\section{1 : Façade de l'ancien hôtel Choudens. Théodore Lambert}

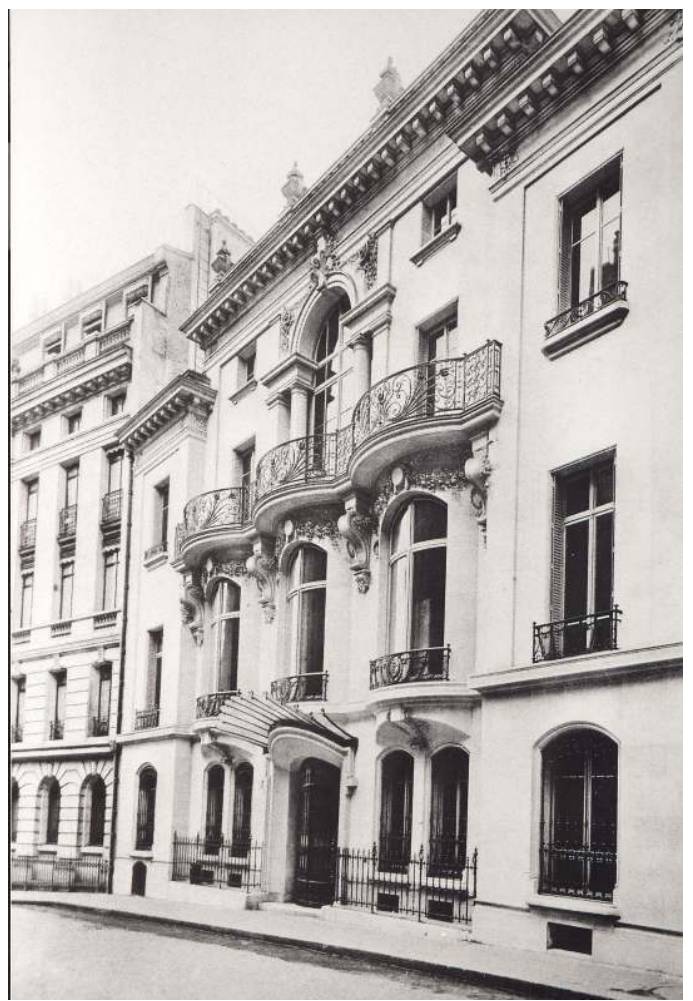

Hôtels privés, plans, façades, coupes, Nouveaux éléments d'architecture, 6e série, Paris, Ch. Schmid éditeur, pl. 30. Bibliothèque des Arts décoratifs, Paris

Cl. A. Chauvin.

\section{Charles Girault, un architecte au sommet de sa gloire}

Charles Girault (1851-1932), élève de Honoré Daumet, Grand-Prix de Rome depuis 1880, est alors au sommet de sa gloire. Selon François Loyer, Charles Girault est le «le plus brillant des architectes "Beaux-Arts" de sa génération, le plus cultivé, sans doute, mais surtout le plus fin [...] et le maître incontesté du style officiel de la Belle Epoque ${ }^{6}$ ». Il a été primé lors du concours de la reconstruction de l'Opéra-Comique après 1887, a construit des immeubles de rapport ${ }^{7}$ et la crypte funéraire de l'Institut Pasteur où repose le savant, en 1896. Mais il est surtout célèbre pour la construction du Petit Palais. Sa notoriété lui 
permettra de construire les tribunes de l'hippodrome de Longchamp en 1903, de travailler pour la famille Rothschild en $1905^{8}$ et surtout de devenir l'architecte du roi Léopold de Belgique ${ }^{9}$. Il entrera à l'Institut en 1902 et deviendra professeur à l'atelier libre de l'École des beaux-arts.

7 Pour ses différents chantiers, Girault choisit des artisans dont il apprécie le travail et reconnaît le savoir-faire. Il leur manifeste une grande fidélité et son « architecture est toujours le faire-valoir de leur œuvre comme si l'un n'allait pas sans l'autre ${ }^{10} »$. La serrurerie de l'hôtel Choudens est exécutée par les établissements Bardin, la menuiserie par M. Perney, la couverture par les établissements Leblond, les staffs par A. Cruchet, la sculpture par Gustave Germain. Les marbres sont fournis par la Société marbrière d'Avesnes. La plupart de ces artisans a collaboré au chantier du Petit Palais ou à celui de la crypte de l'Institut Pasteur ${ }^{11}$.

\section{Un parti architectural traditionnel}

8 Charles Girault construit donc un hôtel, de deux étages et d'un niveau de combles, pour accueillir Paul de Choudens, sa femme et ses trois filles. Lors de l'Exposition universelle de 1900, les esprits avaient été frappés par la discrétion et même par l'absence de couleur des décors. Girault suit ce goût des tonalités claires et utilise des pierres de couleurs blanches, pierre de Comblanchien, pierre de Chauvigny, pierre de Savonnières, pour les différents niveaux de la façade ${ }^{11}$. Les diverses répartitions se lisent tout de suite, grâce au traitement différencié des fenêtres. Les services du sous-sol sont éclairés par de petites fenêtres au ras du sol, isolées de la rue par une grille le long de la façade et un trottoir. Ceux du rez-de-chaussée et la loge du concierge possèdent aussi des baies protégées par des grilles. Les trois fenêtres du premier étage, légèrement incurvées, surmontées de guirlandes, éclairent le salon. Un balcon, aux belles ferronneries suivant les courbes du niveau inférieur, souligne l'emplacement de la salle d'audition. Celle-ci, au centre de la façade, se distingue par une serlienne, de grande ampleur, destinée à éclairer les deux niveaux de la pièce. Une balustrade de pierre ornée de pots à feux dissimule le toit terrasse et la partie du toit mansardé abritant les chambres des domestiques (ill. 2). 


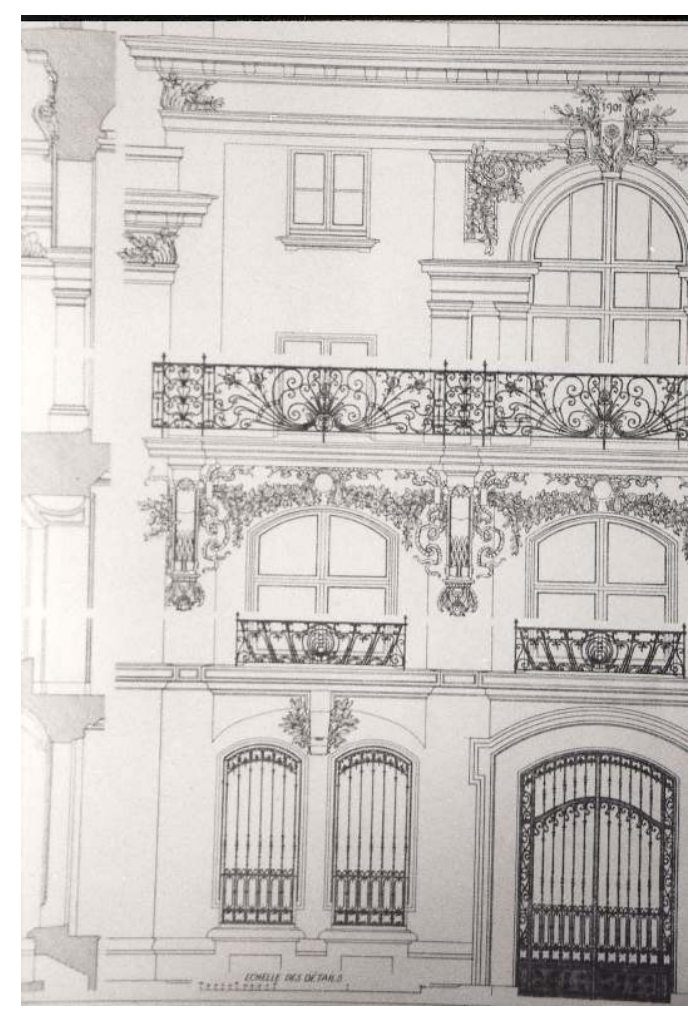

Hôtels privés, plans, façades, coupes, Nouveaux éléments d'architecture, 6e série, Paris, Ch. Schmid éditeur, pl. 30. Bibliothèque des Arts décoratifs, Paris

Cl. A. Chauvin.

9 Paul de Choudens a sans doute défini un programme précis. Girault doit construire la maison d'une famille et celle d'un éditeur de musique où Choudens peut travailler et recevoir beaucoup de musiciens et d'amateurs de musique. L'immeuble comprend une salle d'archives ${ }^{12}$, un grand salon d'audition et un cabinet de travail pour Monsieur ${ }^{13}$.

10 Le plan traditionnel, suit la règle de la répartition tripartite des différents espaces. Au sous-sol se trouvent toutes les installations techniques; les services (cuisine, office et pièces réservées aux gens de maisons et au concierge), la salle des archives à demi enterrée et l'entrée sont au rez-de-chaussée. La partie consacrée à la réception et aux chambres se situe au premier étage. Mais, grâce au dénivelé dû à la pente de terrain, la partie publique (salons, salle à manger, fumoir) se trouve aussi au rez-de-jardin (ill. 3). 


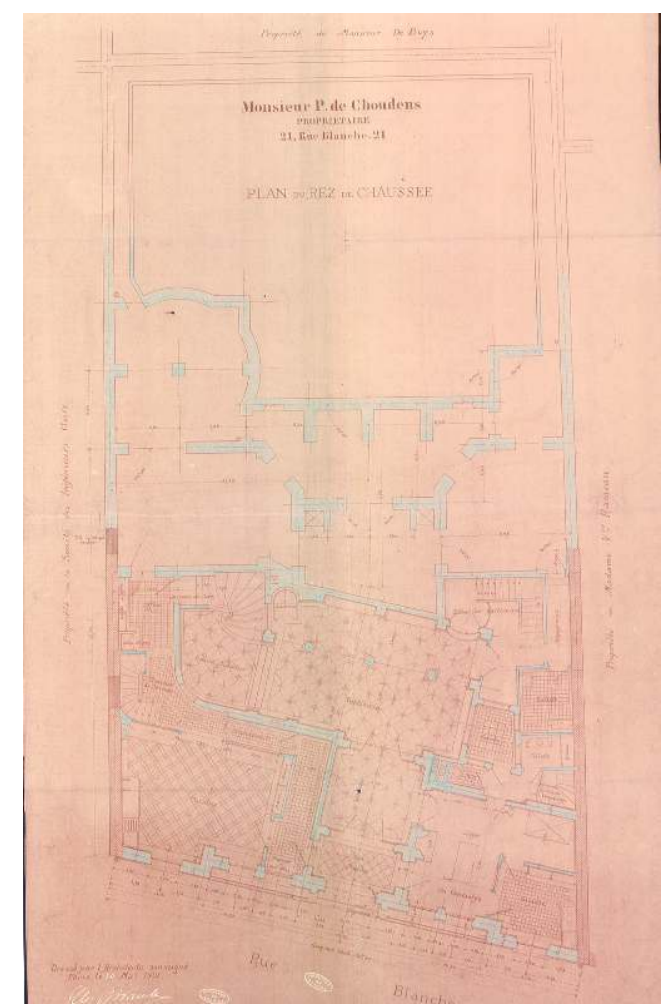

Archives de Paris VO ${ }^{11} 324$

$\mathrm{Cl}$. Archives de Paris.

$11 \mathrm{Au}$ premier étage, Girault a choisi de desservir les pièces d'ostentation par une large galerie. Il conserve la disposition en enfilade reliant les pièces principales (fumoir, grand salon, galerie, petit salon, salle à manger). Les pièces de réception affectent différentes formes, le grand salon est rectangulaire alors que le petit salon est circulaire. Le jardin d'hiver attenant à la salle à manger et ouvrant sur la terrasse offre un espace supplémentaire de réception. Les pans coupés et lignes ondulées suggèrent une différente appropriation de l'espace et agrémentent la façade sur le jardin. Comme au XVIII ${ }^{e}$ siècle, les chambres des maitres de maison se trouvent à l'étage consacré à la réception. Même si l'on peut penser qu'il n'est plus d'usage d'ouvrir sa chambre les jours de réception. La chambre, qui est sans doute celle de "Madame», possède une petite terrasse et un boudoir aux multiples ouvertures sur le jardin. C'est à la fois «une réponse à la valorisation hygiéniste du bon air $^{14}$ » et un agrément supplémentaire pour ces pièces (ill. 4). 


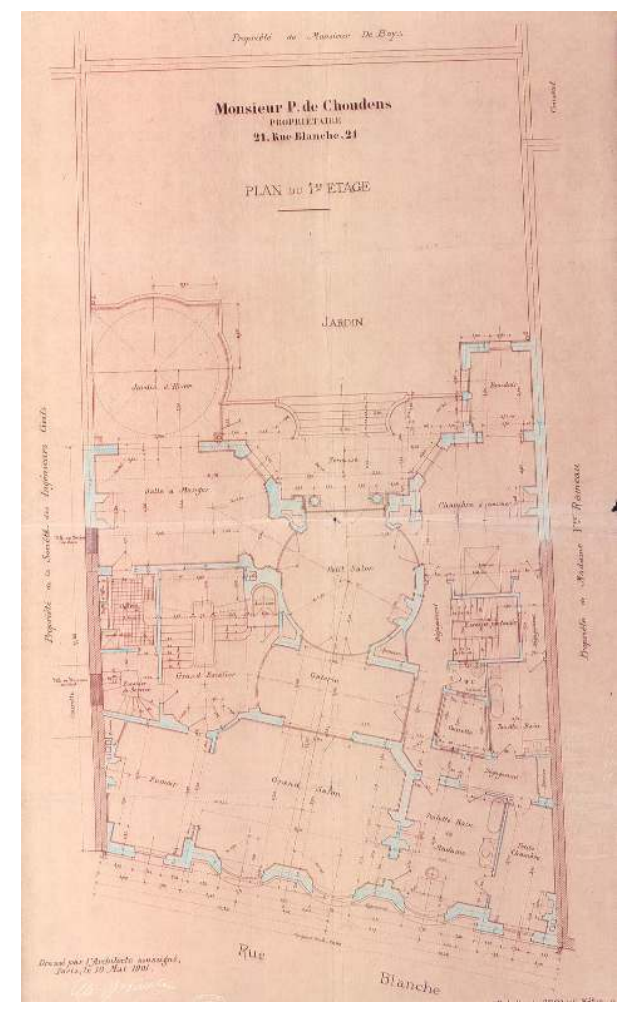

Archives de Paris VO ${ }^{11} 324$

$\mathrm{Cl}$. Archives de Paris.

Une partie du deuxième étage est consacrée aux chambres des enfants et à celle de l'institutrice à proximité de la salle d'étude. Toujours en application du choix de la meilleure orientation, une grande terrasse et une loggia aux colonnes de marbre prolongent les deux chambres principales, donnant sur le jardin. Côté rue, se trouve la salle d'audition qui est la caractéristique de cet hôtel. Très haute de plafond, environ 7 mètres, elle comporte, à mi-étage, une tribune accessible par un petit escalier ${ }^{15}$. Le dernier étage est réservé aux chambres des domestiques.

13 La hiérarchie des différents escaliers est extrêmement lisible : un escalier de service, un grand escalier, un ascenseur qui relie le grand vestibule au premier et au deuxième étages et un escalier de famille, isolé des autres, qui ne dessert que les chambres des parents et des enfants. On remarque aussi la hiérarchie et la grande aisance des espaces de circulation, les dimensions vont décroissant, de la galerie du premier étage aux simples couloirs du dernier étage. Ce système hérité des siècles passés est encore reproduit au tout début $\mathrm{du} \mathrm{XX}^{\mathrm{e}}$ siècle dans les habitations bourgeoises auxquelles il ajoute une référence supplémentaire.

\section{La maîtrise des nouvelles techniques}

14 Au sous-sol se trouvent les caves à bois, à charbon et à vins avec accès direct depuis la rue, une pièce pour le calorifère et un espace pour le monte-charge ainsi que pour la machinerie hydraulique de l'ascenseur. S'y trouvent également le système d'alimentation, de filtrage et de chauffage de l'eau. L'abondance de l'eau est acquise depuis le dernier 
tiers du XIXe siècle dans les habitations luxueuses. Les équipements d'hygiène sont installés sans parcimonie, soit un cabinet de toilette par chambre et une salle de bains par étage. Il faut remarquer que, grâce à la présence de courettes, Girault a permis que salles de bain, cabinets de toilettes et water-closets possèdent des fenêtres.

Pour une meilleure hygiène des pièces, les murs des salles de bain, sont recouverts de lincrusta-walton sur une hauteur d'environ $1 \mathrm{~m} 50^{16}$. Ce matériau résistant aux chocs, absolument imperméable est utilisé depuis une vingtaine d'années. Il se prête à tous les reliefs, ici un décor de natte, peint en blanc ${ }^{17}$.

Le calorifère à vapeur à basse pression est utilisé dans chaque pièce. Toujours soucieux d'améliorer le confort des habitants, Girault complète le système d'un dispositif, placé dans la rosace du plafond, qui corrige la ventilation des pièces de réception et perfectionne le tirage ${ }^{18}$. Les radiateurs sont dissimulés «dans des enveloppes de tôle perforée $^{19}$ » et les bouches de chaleur, décorée de quadrilobes en laiton, sont encore visibles dans le plancher.

Girault a choisi d'installer un ascenseur hydraulique de la marque Piffre dans la cage du grand escalier. Il permet d'atteindre les espaces de réception au premier étage et la salle d'audition au deuxième. L'ascenseur confère à l'hôtel particulier un statut valorisé mais « il doit s'intégrer au grand escalier, au revêtement de marbre [...] et présenter une cohérence avec cet ensemble ${ }^{20}$ ». Aussi, la cage de bois, décorée de guirlandes et aux portes à petits carreaux ressemble beaucoup à une chaise à porteurs ${ }^{21}$.

Le dessin très enlevé de l'escalier principal permet de s'interroger sur sa technique de construction. Girault, comme au Petit-Palais, a-t-il utilisé du béton armé ? Il est un peu difficile de l'affirmer aujourd'hui mais il est possible, malgré l'étroitesse de la façade et le coût d'une telle réalisation pour un particulier, que Girault ait enchâssé un escalier en béton dans un hôtel de construction traditionnelle ${ }^{22}$.

\section{La virtuosité de la décoration}

Grâce à un jeu subtil de références, Girault propose des éléments décoratifs et des motifs architecturaux de différentes époques et influences. Dans le vestibule, la partie vitrée audessus de la porte de la conciergerie évoque la Sécession et les murs sont décorés d'arcs outrepassés. Dans l'escalier et sur les paliers, les arcs et les colonnes jumelées rappellent les édifices romains. Au rez-de-chaussée, les sols de l'entrée et du vestibule sont couverts de carreaux de marbre jaune et blanc qui forment des dessins géométriques. «Ces motifs, inventés par Girault, évoquent ce que cherchent les jeunes architectes européens au début du $\mathrm{XX}^{\mathrm{e}}$ siècle. Des conceptions décoratives très simples, mais originales, fondées sur un nombre réduit d'éléments géométrique ${ }^{23}$.» Les marbres enchâssés dans les lambris d'appui et des colonnes de marbre terre de Sienne répondent aux couleurs du pavement.

De grands panneaux de marbre violet, en harmonie avec les colonnes du premier étage et la mosaïque des paliers, couvrent les murs de la cage d'escalier. Le grand escalier possède une remarquable rampe de style Louis XV. La finesse de son dessin ainsi que les volutes agrémentées de boutons de fleurs rappellent la rampe de l'immeuble de la place Laborde et celle du Petit Palais. La petite porte devant la cage de l'ascenseur décorée de tiges sinueuses et de d'iris, est proche de l'Art nouveau (ill. 5). 


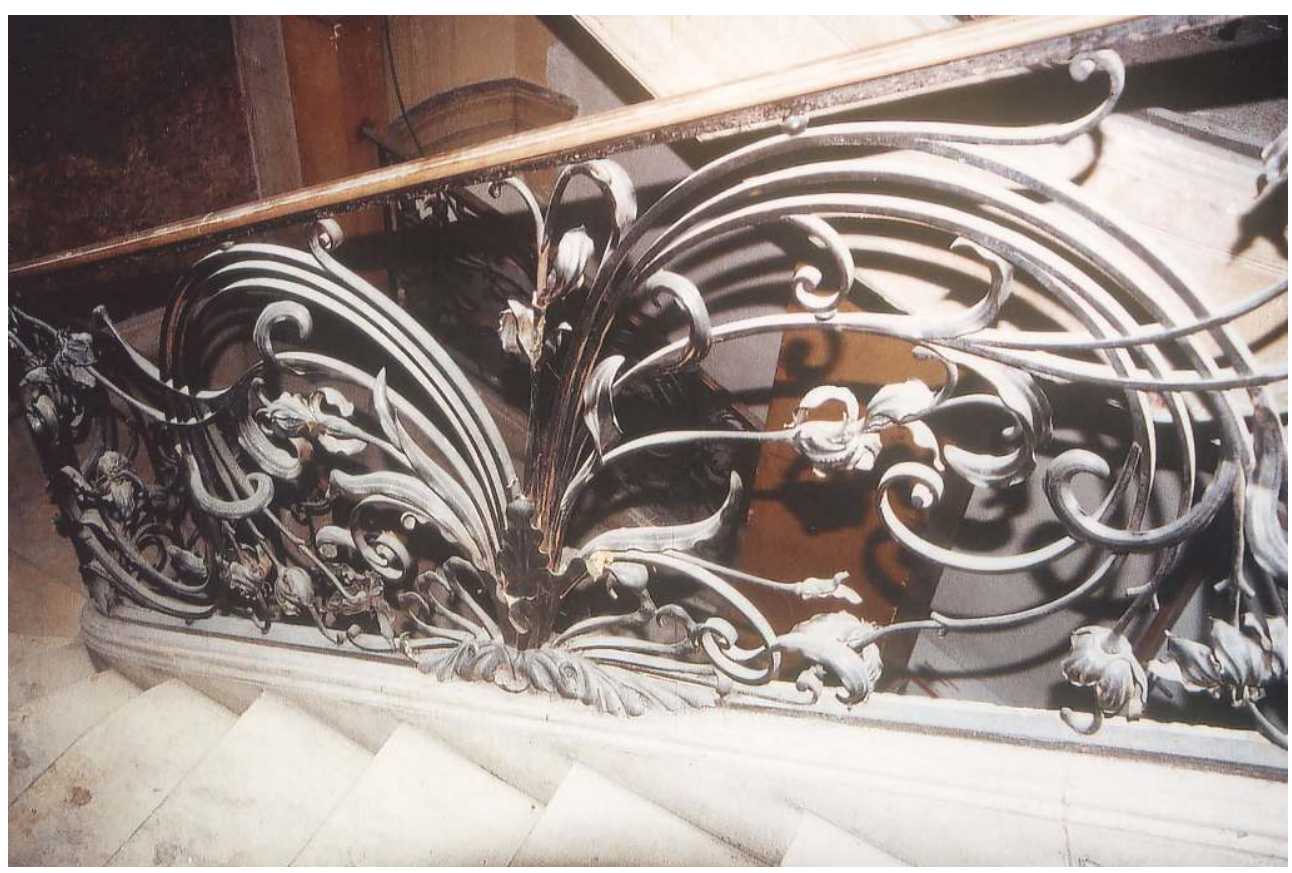

Cl. A. Chauvin

La porte de fer forgé qui sépare le palier du premier étage de la galerie possède la même qualité et les mêmes formes souples et sinueuses que la rampe d'escalier. Le motif est constitué d'un décor de tiges dont certaines se terminent par des fleurs en cuivre alors que d'autres prennent la forme d'une plume de paon et d'œillets mongols. Des verres de couleur enchâssés dans le métal pour figurer les couleurs de cet oiseau donne un aspect exotique et précieux à cette porte qui ouvre sur l'espace de réception. L'imposte, plus sobre, fait penser à la ferronnerie espagnole traditionnelle.

Le plafond de la salle d'audition est formé d'un lambris de chêne aux rehauts d'or. Le mauvais état actuel du plafond permet de savoir qu'il s'agit d'un décor de staff qui reproduit les différents détails d'un décor à caissons de style Renaissance. Plus anecdotique mais bien représentative de la vie mondaine de la Belle Époque, est la présence de miroirs placés sur les volets du grand salon. Une fois ceux-ci fermés, les fenêtres sont transformées en grandes glaces et procurent une ambiance festive les soirs de réception tout en permettant d'agrandir l'espace ${ }^{24}$.

\section{L'école de la rue Blanche}

En 1943, le centre de jeunesse du spectacle, centre de formation professionnelle pour jeunes gens et jeunes filles, dont l'enseignement est éparpillé dans différents bâtiments du $17^{\mathrm{e}}$ arrondissement, s'installe dans l'hôtel Choudens ${ }^{25}$. Rien n'est fait pour transformer cette demeure familiale en école avant avril 1951, date de l'inauguration du théâtre dont on peut supposer qu'il s'installe dans la salle d'audition. Cette école qui a porté différents noms $^{26}$ a été, le plus souvent, appelée "l'école de la rue Blanche». Elle assure la préparation au Conservatoire national et forme des décorateurs et des costumiers ${ }^{27}$. 

moment où l'école devient un lycée technique d'État. L'immeuble se transforme peu à peu pour accueillir les différents ateliers de couture et de décoration, les loges, les salles de cours et l'administration. Devenue École nationale supérieure des arts et techniques du théâtre en 1993, l'école manque cruellement de place et choisit de déménager à Lyon en 1998. L'immeuble est désaffecté à partir de cette date. Le 9 mai 1980, les façades et les toitures, l'escalier intérieur avec sa rampe en fer forgé et le jardin d'hiver avaient été inscrits au titre des monuments historiques.

Cet hôtel fait partie des derniers hôtels construits à Paris dans un style historique. Charles Girault y montre son érudition mais évite les outrances on ne peut que remarquer l'élégance du plan, l'excellence des proportions et l'exécution extrêmement soignée de tous les décors. Il peut paraître au premier abord bien classique, mais comme d'autres œuvres de Girault, il se trouve en fait bien loin des cadres établis. Les travaux de restauration engagés pour la nouvelle affectation de cet immeuble permettront de retrouver les tons d'origine de la façade, les couleurs des huisseries et la dorure des ferronneries et des pots à feu. Ils participeront à la redécouverte de cet architecte, reconnu depuis la réouverture du Petit Palais et qui mériterait comme l'écrit Claude Mignot de «retrouver sa place, une des premières dans le panthéon des architectes français».

\section{NOTES}

1. Je dédie cet article à la mémoire de ma mère ( $\$ 1998)$, ancienne élève de l'École CésarFranck et du centre national de préparation au certificat d'aptitude de l'enseignement musical (C.A.E.M.) du lycée La Fontaine à Paris. Je remercie tout spécialement mon mari, Denis Génuit, qui a attiré mon attention sur la façade de cet immeuble. Je remercie également, Bernard Brossollet, directeur général des éditions Choudens, pour son aide précieuse tout au long de mes recherches et Claire Gobert pour sa relecture attentive.

. Anick Devriès et François Lesure, Dictionnaire des éditeurs de musique français, vol. 2 (1820-1914), Genève, éditions Ninkoff, 1988.

2. . En 1891, Choudens publie Valse romantique, Rêverie, Ballade, Marche écossaise et Tarantelle styrienne. Dans la seconde moitié du XXe siècle, la maison Choudens a publié les œuvres de Marcel Landowski et d'Henri Sauguet ainsi que de nombreuses musiques de films dont plus récemment celle des Choristes. La maison est restée indépendante jusqu'en 2006.

3. . La maison d'édition est situé 30, boulevard des Capucines (II arr.) entre 1887 et 1925.

4. . Arch. dép. Seine $\mathrm{D}^{1} \mathrm{P}^{4} 126$ calepin du cadastre de 1876 et $\mathrm{DQ}^{18} 1369$, la vente est faite à Paul de Choudens et Marie Polge, sa femme, le 12 septembre 1899 devant Maitre Cottenet.

5. . Arch. dép. de la Seine VO ${ }^{11} 3324$.

6. . François Loyer, Histoire de l'architecture française, de la Révolution à nos jours, Paris, Mengès, 1999, p. 183.

7. . Immeuble de rapport 12 bis, place Laborde (aujourd'hui place Henri Bergson) à Paris $8^{e}$ arr., en 1889 ; 36, avenue Henri-Martin. (où Girault habitait, aujourd'hui avenue Georges Mandel) à 
Paris $16^{\mathrm{e}}$ arr., en 1896 et l'hôtel particulier d'un artiste 7, place des États-Unis à Paris, $16^{\mathrm{e}}$ arr., en 1893. Voir A. Louvet, « Charles Girault, membre de l'Institut », L'Architecture, 1933, p. 253-262.

8. . Il est sollicité par Édouard de Rothschild pour construire le manoir de Sans-Souci à Gouvieux, voir Pauline Prevost-Marcilhacy, Les Rothschild, bâtisseurs et mécènes, Paris, Flammarion, 1995.

9. Agrandissement château de Laeken en 1903, Arcades du jardin du Cinquantenaire en 1904, achèvement du palais du Congo en 1911, à Bruxelles.

10. . François Loyer, op. cit., Paris, Mengès, 1999, p. 402.

11. . Louis-Charles Boileau, « Causeries, art et pratique », L'Architecture, 16 e année, 3 janvier 1903, $\mathrm{n}^{\circ}$ 1, p. 6. Voir Gilles Plum, Le Petit-Palais, chef d'œuvre de Paris 1990, Paris, Paris-Musées, 2005, p. 245-247 pour les artisans ayant travaillé au Petit-Palais.

12. . On peut se demander pourquoi une si grande salle d'archives a été construite. N'aurait-elle pas eu sa place plutôt dans l'immeuble du boulevard des Capucines?

13. . Dans le permis de construire (Arch. dép. Vo ${ }^{11}$ 3324) au premier étage, se trouve une chambre et une salle de bain à droite du grand salon remplacée par « un cabinet de travail pour Monsieur » dans Louis-Charles Boileau, ibid., p.3. Notons qu'il ya très peu de différences entre le permis de construire et la réalisation.

14. . Monique Eleb et Anne Debarre, L'Invention de l'habitation moderne 1880-1914, Paris, 534 p., p. 274.

15. . Arch. nat. 285 AP 15. Girault a étudié le plan de salles de concert à Paris, Bayreuth, Bruxelles et Monte-Carlo, et a sans doute réuni une documentation sur ce sujet comme le faisait cette génération d'architectes pour mieux appréhender les commandes de leurs clients. Rappelons que Girault a été primé lors du concours de l'Opéra Comique. Peut-être est-ce à cette occasion que Girault et Choudens se sont rencontrés.

16. . Louis-Charles Boileau «Causeries, art et pratique », op.cit., p. 6.

17. Musée des arts décoratifs, département des papiers peints, voir le Catalogue de la compagnie française de lincrusta-walton, décor de natte, p.11.

18. . La Construction moderne, avril 1895, p.358-359.

19. . Louis-Charles Boileau, « Causeries, art et pratique », op. cit., p. 3.

20. . Monique Eleb et Anne Debarre, op.cit., p. 407.

21. . Une cage d'ascenseur du même style est déposée dans le vestibule de l'immeuble du 36, avenue Georges-Mandel où habitait Girault.

22. . Je remercie Gwenaël Delhumeau, enseignant à l'école d'architecture de Versailles, pour ses précisions.

23. . Gilles Plum, op.cit., p.108.

24. . Cette disposition est toujours en place. On la retrouve dans d'autres hôtels particuliers, par exemple dans un des salons de l'hôtel Amelot de Gournay à Paris.

25. . BnF, département des arts du spectacle, fonds de l'École nationale supérieure des arts et techniques du théâtre (E.N.S.A.T.T.), $4^{\circ}$ COL. 60, pièce 13.

26. Centre d'apprentissage public du spectacle avant 1957 puis centre d'enseignement technique d'art dramatique en 1967.

27. . Voir le documentaire d'Anne-Marie Lallement On l'appelait l'école de la rue Blanche (1997), Forum des Images, cote 11432 


\section{RÉSUMÉS}

Paul de Choudens, à la tête de la maison d'édition qui a publié Berlioz et Bizet, fait construire, en 1901, un hôtel particulier dans l'ancien quartier de la Nouvelle-Athènes à Paris. Il choisit l'architecte du Petit-Palais, Charles Girault, alors au sommet de sa gloire. Girault construit une demeure familiale selon le plan traditionnel des demeures aristocratiques aux répartitions tripartites habituelles (services aux sous-sol et au rez-de-chaussée, partie publique au premier étage et partie privée au deuxième) mais aussi un lieu de travail et de réception puisque le programme comporte une salle d'archives et une salle d'audition. Derrière une façade représentative de la «Belle Époque », l'hôtel possède tous les éléments du confort le plus récent ainsi que des éléments décoratifs de grande qualité. Longtemps occupé par la célèbre école de théâtre de la rue Blanche, puis désaffecté, le bâtiment va retrouver une nouvelle destination culturelle.

Paul de Choudens, as head of a publishing house behind the works of Berlioz and Bizet, had a townhouse built in 1901 in the old Paris quarter known as "Nouvelle-Athènes" (New Athens). He chose as architect Charles Girault, the designer of the Petit-Palais, who was at the time at the peak of his glory. Girault built a family dwelling based on the traditional blueprint of aristocratic homes, with the usual three sections (service area in the basement, public area on the second floor and the private dwelling on the third floor), but also with a work space and reception area because the layout included an archives room and an auditorium. Behind a facade representative of "La Belle Epoque", the townhouse contains all the most modern comforts as well as finequality decorative elements. Long inhabited by the famed dramatic school of rue Blanche, afterwards unused, the building will now become a new cultural destination again.

Paul de Choudens, Leiter des Musikverlags, der Berlioz' und Bizets Werke veröffentlichte, ließ sich 1901 ein Hôtel particulier (Stadtpalais) in dem sogenannten Pariser Stadtteil der NouvelleAthènes bauen. Er beauftragte damit Charles Girault, den damals hochgepriesenen Architekten des Petit-Palais. Girault baute ein Familienhaus, der dem gewohnten dreiteiligen Plan der aristokratischen Wohnsitze entsprach : so Diensträume im Unter- und Erdgeschoss, öffentliche Räume in dem ersten Stock und Privaträume in dem zweiten. Er plante auch Arbeits- und Empfangsräume, ebenso wie Archiv- und Hörsaal. Hinter seiner Fassade im Belle époque Stil bietet das Hôtel alle Bestandteile der modernsten Ausstattung, ebenso wie Ornamente höchster Qualität. Es beherbergte jahrelang die Theaterschule der rue Blanche, wurde dann leergestellt, wird aber bald einem neuen kulturellen Zweck gewidmet.

\section{AUTEUR}

\section{AGNÈS CHAUVIN}

Agnès Chauvin a soutenu une maîtrise d'histoire de l'art sur La cathédrale de Dax sous la direction de Daniel Rabreau à l'université de Bordeaux III puis un D.E.A sur Les hôtels particuliers du second Empire d'après la presse architecturale de l'époque sous la direction de Bruno Foucart et Françoise Hamon à l'université de Paris IV. Intéressée par la période des années trente, elle a publié un 
article sur un piano Érard-Ruhlmann dans L'Estampille-L'Objet d'art en mars 1998 et un article sur l'immeuble de presse de la rue du Louvre dans Livraisons d'histoire de l'architecture en janvier 2006. Elle est documentaliste-recenseur à la DRAC Ile-de-France depuis 2000. Adresse électronique : agnes.chauvin@culture.gouv.fr 